\title{
Multisensorsysteme für Einweg-Bioreaktoren
}

\author{
Steffen Henkel ${ }^{1}$, Sascha Beutel ${ }^{1}$, Christian Endres $^{2}$, Daniel Riechers ${ }^{2}$, Thomas Scheper ${ }^{1}$ \\ ${ }^{1}$ Institut für Technische Chemie, Leibniz Universität Hannover, Callinstraße 5, 30167 Hannover \\ ${ }^{2}$ Sartorius Stedim Biotech GmbH, August-Spindler-Straße 11, 37079 Göttingen
}

\section{Zusammenfassung}

Zur Überwachung biotechnologischer Prozesse in Einweg-Biorektoren bedarf es einer adaptierbaren Sensorik. Ziel der hier vorgestellten Arbeiten ist die Testung relevanter Sensorkonzepte und die Konstruktion eines kompletten Adaptermoduls zur Aufnahme prozesstechnisch relevanter Größen während einer Kultivierung. In einzelne Stufen des Entwicklungsprozesses von der Auswahl über Tests an den Einzelkomponenten bis hin zu ersten Tests des Prototypen soll hierbei ein Einblick gewährt werden.

\section{Einleitung}

Die biotechnologische Herstellung und Vermarktung rekombinanter Produkte erlebt ein seit Jahren ungebrochenes weltweites Wachstum. Hierbei geht der Trend weg von teuren und unflexiblen monolithischen Produktionsstraßen - hin zu flexiblen, vorsterilisierten, skalierbaren, einmal verwendbaren Systemen. Die zunehmende Verbreitung von Einwegsystemen in der pharmazeutischen Industrie resultiert aus vor allem aus dem geringeren Validierungsaufwand beim Einsatz dieser Systeme. Konventionelle Edelstahlreaktorsysteme und Medien- bzw. Produkthaltetanks müssen nach jedem Produktionszyklus zeitaufwändigen Reinigungs- und Sterilisierungszyklen mit hohem Dokumentationsaufwand unterworfen werden. Einwegsysteme bieten demgegenüber den Vorteil, dass sie als ready-to-use-Lösung schnell in Betrieb genommen und anschließend unkompliziert entsorgt werden können, wodurch sich Produktionszyklen deutlich verkürzen lassen. Die Sterilisationsvalidierung verlagert sich hierbei zum Zulieferer, die Reinigungsvalidierung entfällt. Der reduzierte Zeitaufwand ist vor allem bei der Entwicklung pharmakologischer Wirkstoffe vorteilhaft, da die time-to-market in vielen Fällen erfolgsentscheidend ist. Ein weiterer Vorteil von Einwegsystemen besteht in dem signifikant niedrigeren Risiko der Kreuzkontamination zwischen verschiedenen Prozessen. Aus wirtschaftlicher Sicht führt die Verwendung von Einwegprozessequipment $\mathrm{zu}$ niedrigeren initialen Anschaffungskosten und einer günstigeren Kostenverteilung über die Projektlaufzeit. Aufgrund der variablen Anbindung von Einwegsystemen an beispielsweise Medienanschlüsse oder die auf die Produktion (Upstream) folgenden Downstreamingschritte ergeben sich flexiblere Prozessstrukturen als bei festverrohrten Produktionsstraßen.

Um eine gleichbleibende hohe Qualität bei der Produktion pharmakologischer Wirkstoffe zu gewährleisten, werden in Kombination zu den single use Bioreaktorsystemen verlässliche Sensorsysteme benötigt, die optimal in diese Systeme migrieren. Durch die PAT-Initiative (Process Analysis Technology) der FDA, welche den gezielten Einsatz von Prozessanalysentechnik in der Entwicklung, Produktion und dem Qualitätsmanagement in der Nahrungsmittel- und der pharmazeutischen Industrie fördert, wird die Bedeutung der Sensorik für Bioprozesse in den kommenden Jahren weiter zunehmen. Auch die Einwegsensoren und Einwegreaktorsysteme werden sich an den strengen Standards der FDA messen lassen müssen.

\section{Sensorkomponenten}

Da es wirtschaftlich nicht sinnvoll ist, das Messsystem nach der Nutzung komplett zu entsorgen, ist es notwendig, das Messsystem in einen günstigen medienberührenden Einwegteil mit innerer Sterilbarriere, 
sowie einen an das Einwegteil koppelbaren wiederverwendbaren Teil (Transmitter) aufzutrennen. In der Planungsphase mussten hierfür die Prozessgrößen definiert und geeignete Technologien ausgewählt werden.

\subsection{Auswahl}

Für den Einsatz in Einweg-Bioreaktoren sollten geeignete Sensortechnologien ausgewählt werden. Gesichtspunkte, die bei der Auswahl berücksichtigt wurden, waren, neben der preislichen Umsetzbarkeit, die Verfügbarkeit und die Messgenauigkeit. Zudem mussten die zu detektierenden relevanten Prozessgrößen ausgewählt werden. Hierbei fiel die Entscheidung auf den $\mathrm{pH}$, den Gelöstsauerstoff, die elektrische Leitfähigkeit sowie die Temperatur. Während zur Temperaturmessung auf einen Platinthermistor des Typs Pt1000 und zur Messung der elektrischen Leitfähigkeit auf Dickschichtelektroden zurückgegriffen wurde, werden zur Messung von $\mathrm{pH}$ und Gelöstsauerstoff optische Chemosensoren verwendet.

\subsection{Funktionsprizip der optischen Chemosensoren}

Der optische Gelöstsauerstoff-Sensor besteht aus einem sauerstoffsensitiven Fluoreszenzfarbstoffen, der in einer Silikonmatrix immobilisiert vorliegt. Zur Herstellung des optischen pH-Sensors wurde der Fluoreszenzfarbstoff Dihydroxypyrensulfonsäure kovalent in einem Polyethylenglycolhydrogel immobilisiert. Die eigentliche Messung der verschiedenen Parameter basiert dabei auf unterschiedlichen Prinzipien.

Die Messung des pH-Wertes erfolgt durch eine ratiometrische Messung. Dabei wird unter Anregung bei $405 \mathrm{~nm}$ mittels einer geeeigneten LED die Fluoreszenzintensität bei den zwei Maxima des kontinuierlichen Emissionspektrums gemessen und das Intensitätsverhältnis berechnet. Während die Fluoreszenzintensität des Maximums bei $505 \mathrm{~nm}$ nicht durch den $\mathrm{pH}$-Wert beeinflusst wird, verändert sich die Fluoreszenzintensität des Maximums bei $445 \mathrm{~nm}$ in Abhängigkeit vom pH (Bild 1). Durch die ratiometrische Messung werden Fehler minimiert, die durch Photobleaching oder variable Abstände des Chemosensors zur Lichtquelle auftreten.

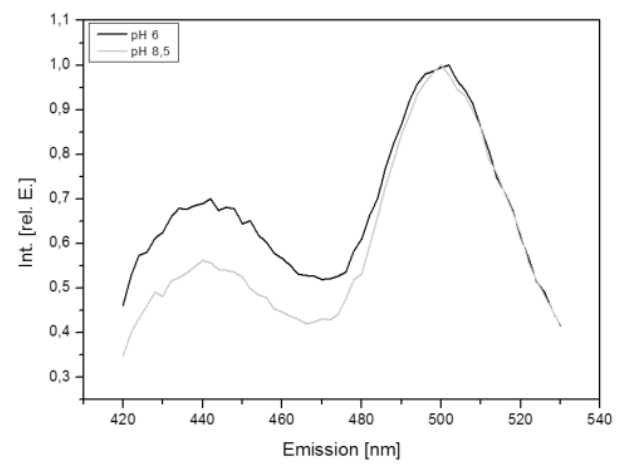

Bild 1 Emissionsspektrum des pH-Sensormaterials bei Anregung @405 nm

Die Messung des Gelöstsauerstoffs erfolgt durch Bestimmung der Fluoreszenzlebenszeit. Da die Fluoreszenz durch Sauerstoff gequencht wird nimmt die Lebenszeit mit zunehmendem Sauerstoffgehalt in der Lösung ab. Dieser Zusammenhang wird durch die Stern-Vollmer-Gleichung beschrieben:

$$
\frac{I_{0}}{I}=\frac{\tau_{0}}{\tau}=1+K_{S V} \cdot[Q]
$$

$\begin{array}{ll}I_{0} \tau_{0} & \text { Fluoreszenzintensität/Fluoreszenzlebenszeit für }[\mathrm{Q}]=0 \mathrm{~mol} / \mathrm{L} \\ I / \tau & \text { Fluoreszenzintensität/Fluoreszenzlebenszeit für [Q] } \neq 0 \mathrm{~mol} / \mathrm{L} \\ K_{S V} & \text { Stern-Volmer-Konstante } \\ {[Q]} & \text { Quencherkonzentration }\end{array}$




\subsection{Integration der Komponenten}

Für die Konstruktion eines Multisensoradapters ist eine modulare Bauweise von Einweg-Sensor und wiederverwendbarer Ansteuerungs-/Detektionseinheit notwendig. Während die elektronisch ausgelesenen Sensoren über einfache Steckverbindungen an die Elektronik angeschlossen werden, wurden für die optischen Chemosensoren Steckverbindungen konstruiert, die durch ihre Form zum einen eine feste Verbindung mit dem Messmodul herstellen, zum anderen aber auch einen definierten Abstand der Faseroptik vom Chemosensor gewährleisten. Sämtliche Sensoren wurden in einem Bauteil vereinigt (Bild 2) und auf ihre Handhabung getestet.

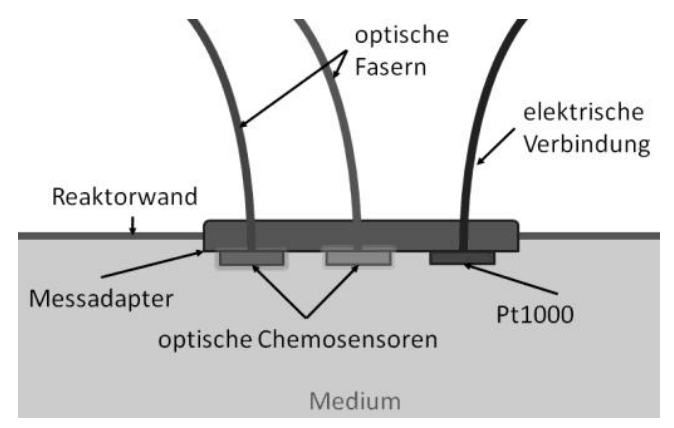

Bild 2 Schematische Darstellung eines Multisensoradapters

\section{Tests der Sensorkomponenten}

Der folgende Abschnitt soll einen kurzen Einblick zu den Eigenschaften ausgewählter Sensorkomponenten bieten. Das Hauptaugenmerk liegt dabei auf den Chemosensoren.

\subsection{Kalibrationsroutine und Bleaching des pH-Chemosensors}

Die Kalibrationsroutine des $\mathrm{pH}-$ Chemosensors folgt einer sigmoiden Boltzmann-Funktion (Bild 3). Es ist zu erkennen, dass die höchste Auflösung im Bereich von pH 6,7 - 8,0 erreichbar ist. In den äußeren Bereichen ist eine geringere Messgenauigkeit zu erwarten, da kleine Änderungen des Intensitätsverhältnisses bereits großen Änderung des pH-Wertes entsprechen.

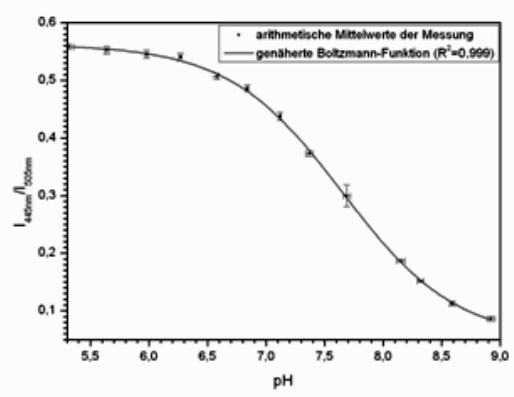

Bild 3 Kalibrationsroutine des $\mathrm{pH}-\mathrm{Chemosensors}$

Die im Chemosensor immobilisierten Fluoreszenzfarbstoffe unterliegen dem Effekt des Photo-Bleaching, d.h. sie bleichen mit zunehmender Bestrahlungsdauer aus. Zwar lässt sich dieser Effekt durch die ratiometrische Messung verringern, doch trotz allem weisen beide Farbstoffe nicht exakt das gleiche Bleichverhältnis auf. Wird zur Überwachung eines Bioprozesses beispielsweise das Messintervall auf drei Minuten festgelegt, so konnte gezeigt werden, dass während der ersten 5.000 Messungen die Abweichung zur Referenzelektrode unter einer Zehntel pH-Einheit lag (50 mM Kaliumphosphat-Puffer $\mathrm{pH}$ 7,4; $150 \mathrm{mM}$ Ionenstärke), was bei dem genannten Messintervall einer Prozessdauer von über zehn Tagen entspricht. 


\subsection{Test des Chemosensors für Gelöstsauerstoff}

Um die Messgenauigkeit des Chemosensors für Gelöstsauerstoff zu prüfen, wurde ein Stufenprofil durchfahren. In Bild 4 lässt sich der Verlauf der Messung nachvollziehen. Im Bereich hohen Sauerstoffgehalts konnte ein starkes Rauschen des Messsignals beobachtet werden. Da die Fluoreszenz durch den anwesenden Sauerstoff gequencht wird, hat dies ein geringes Signal zur Folge, bei dem es zu den Messungenauigkeiten kommt. Mit sinkendem Sauerstoffgehalt nimmt das Signal zu und das Rauschen ab. Im Bereich niedrigerer Sauerstoffkonzentrationen lassen sich mit dem Chemosensor folglich exaktere Messdaten gewinnen.

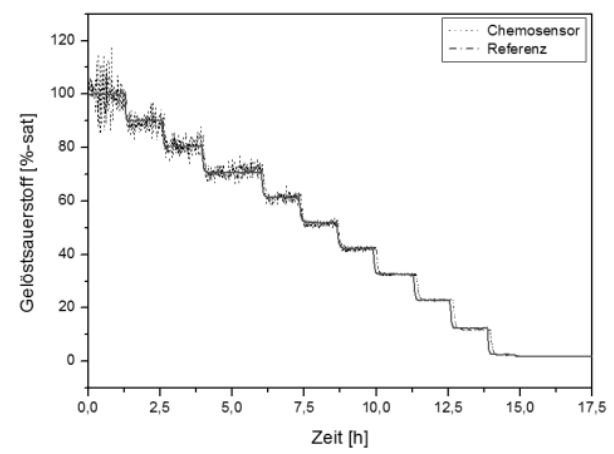

Bild 4 Stufenprofil der Sauerstoffsättigung, nachvollzogen mit Chemosensor und Referenz

\section{Erste Tests des Messadapters}

Nach der Testung der einzelnen Sensortechnologien wurden die Einzelsensoren zu einem Messadapter zusammengefügt. Um die Sensoren in realen Einsatzbedingungen zu testen, wurde der Adapter zur Überwachung einer Kultur von Escherichia coli K12 in TB-Medium eingesetzt. Zur Demonstration der Einsetzbarkeit des Adapters sind in Bild 5 ausgewählte Sensoren gemeinsam mit ihrer Referenz aufgetragen. Während der Kultivierung wurde das Medium mit einer Mischung aus 50\% Druckluft und 50\% Stickstoff begast. Es ist zu erkennen, dass nach 2,5 h der Sauerstoffbedarf der Bakterien rapide ansteigt und so der Sauerstoffgehalt auf 0\% abfällt. Dies ist sowohl am Chemosensor als auch an der Referenz nachvollziehbar. Die Temperatur nimmt im Verlauf der Kultivierung leicht zu. Auch hier zeigt sich, dass beide Temperaturfühler nahezu deckungsgleich verlaufen. Auch für die nicht abgebildeten Sensoren konnte eine gute Korrelation gefunden werden. Die Messwerte für die optische Dichte wurden zur Überwachung des Zellwachstums offline entnommen.

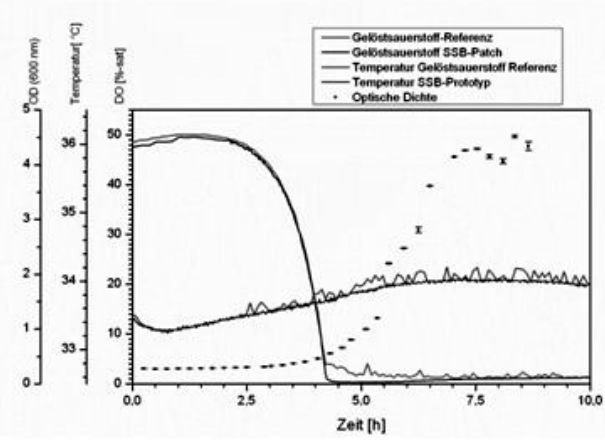

Bild 5 Ausgewählte Messdaten einer E.coli K12 Kultivierung

\section{Ergebnisse}

Die im Sensoradapter verwendeten Technologien eignen sich zur Überwachung von biotechnologischen Prozessen. Da es sich ohne Ausnahme um Einwegsensoren handelt, besitzt der Adapter das Potential die Prozessüberwachung in Einwegsystemen zu verbessern. 